\title{
Lack of relation between venous plasma total catecholamine concentrations and ventricular arrhythmias after acute myocardial infarction
}

\author{
R C STRANGE, M J ROWE, M F OLIVER
}

British Medical fournal, 1978, 2, 921-922

\section{Summary and conclusions}

Electrocardiographic tracings were recorded continuously to monitor ventricular tachycardia and $R-o n-T$ and $R$-on-apex- $T$ ventricular premature beats, and repeated estimations of venous plasma total catecholamine concentrations were carried out in 26 patients admitted to a coronary care unit with acute myocardial infarction. No relation existed between the increased catecholamine concentrations found in these patients and the incidence of ventricular arrhythmias occurring six to 48 hours after the onset of symptoms.

\section{Introduction}

Plasma catecholamine concentrations are raised within the first hours after the onset of acute myocardial infarction ${ }^{1}$ and, together with urinary catecholamine concentrations, may remain raised for several days. ${ }^{2-5}$ Previous studies have related high urinary and plasma catecholamine concentrations to the severity of infarction ${ }^{5} 6$ and the risk of developing serious ventricular arrhythmias. ${ }^{78}$ Correlations between raised plasma adrenaline concentrations and ventricular arrhythmias ${ }^{3}$ and between plasma noradrenaline concentrations and the incidence of arrhythmias ${ }^{9}$ have been reported. We have studied these associations in greater detail by measuring plasma total catecholamine concentrations serially within hours of the onset of symptoms of acute myocardial infarction and by making simultaneous continuous recordings of the electrocardiogram (ECG) and analysing the rhythms with a computer. We report here the results.

\section{Patients and methods}

We studied 26 unselected patients, who formed part of a control group in a study designed to test the effect on arrhythmias of antilipolytic treatment. ${ }^{10}$ All were admitted to the coronary care unit of the Royal Infirmary within 12 hours of the onset of severe central chest pain, the mean time of admission being 5.3 (range 1.25-11.25) hours. The clinical diagnosis of acute myocardial infarction was confirmed in all patients by electrocardiographic changes sufficient to warrant allocation to group 1.1 or 1.2 of the Minnesota code ${ }^{11}$ and by an increase in serum creatine kinase activity. ${ }^{12}$ We excluded from the study diabetics; patients with cardiac failure or cardiogenic shock; and those taking $\beta$-adrenergic blocking agents, methyldopa, and drugs affecting lipid metabolism. During the study patients were given morphine, diazepam, digoxin, diuretics, and other drugs when indicated, but none received lignocaine.

Blood obtained by antecubital venepuncture was collected into

\section{Royal Infirmary, Edinburgh EH3 9YW}

R C STRANGE, BSC, PHD, lecturer, department of clinical chemistry

M J ROWE, MD, FRCPED, research fellow, department of cardiology (present appointment: senior registrar in geriatrics, Royal Victoria Hospital, Edinburgh)

M F OLIVER, MD, FRCP, professor of cardiology, departments of cardiology and medicine 10-ml plastic tubes containing heparin and sodium metabisulphite $(5 \mathrm{mg})$. Samples were taken on admission to the study, one hour later, and at 4,12,20,24,32, and 40 hours after admission. All blood samples were centrifuged for three minutes at $2500 \mathrm{~g}$ within three minutes of removal. Plasma $(5 \mathrm{ml})$ was then added to glassstoppered tubes containing $1 \mathrm{ml}$ of perchloric acid $(4 \mathrm{~mol} / \mathrm{l})$, and after mixing and five minutes' centrifugation at $2500 \mathrm{~g}$ the clear supernatant was removed. Total catecholamine concentrations were estimated by a fluorimetric trihydroxyindole method, with the precautions recommended by Carruthers et al. ${ }^{13}$ Recovery of known concentrations of adrenaline and noradrenaline from plasma was $84 \cdot 0 \pm \mathrm{SD} 7 \cdot 1 \%$.

The ECG was recorded on continuous magnetic tape for 48 hours from the start of the study. The record was then replayed at 60 times normal speed for visual scanning on a 10-line raster display, and the actual numbers of ventricular premature beats (VPB) and of episodes of four or more consecutive VPB were counted. All episodes of four or more consecutive VPB were printed on paper for further interpretation and were classified as ventricular tachycardia if the overall rate was above $100 /$ minute. A special-purpose hybrid computer was used when the VPB occurred too frequently for visual counting. ${ }^{14}$ The computer was programmed to detect separately all VPB with a coupling interval of $400 \mathrm{~ms}$ or less, and these were printed on paper to locate $R$-on-T and R-on-apex-T wave phenomena. While the computer scan was in progress the ECG was also visually displayed to provide a check on the signals counted by the computer and to locate $\mathrm{R}$-on- $\mathrm{T}$ phenomena with a coupling interval of more than $400 \mathrm{~ms}$.

\section{Results}

PLASMA TOTAL CATECHOLAMINE CONCENTRATIONS

All plasma total catecholamine concentrations in 25 of the 26 patients studied were above the reference range $(0 \cdot 2-0.5 \mathrm{mg} / \mathrm{l})$ for up to $\mathbf{4 0}$ hours after the onset of symptoms. The values varied widely between patients, though in each individual patient they were fairly constant throughout the study. The table shows the distributions of the mean and maximum plasma catecholamine concentrations found in the patients.

\section{VENTRICULAR ARRHYTHMIAS}

Ventricular tachycardia-Of the 26 patients, 14 had ventricular tachycardia. Twenty episodes were recorded in the first 12 hours of the study, 27 episodes between 12 and 24 hours, and only three episodes between 24 and 48 hours after the study began.

$V P B$ of the R-on-T type-Sixteen patients showed VPB of the $R$-on-T type: these included VPB in which ectopic $R$ waves interrupted $T$ waves of sinus beats and also those in which ectopic $R$ waves interrupted $T$ waves of preceding VPB. In the first 12 hours after the onset of symptoms there were 19 episodes of sinus $T$-wave interruption and 23 episodes of ventricular ectopic $T$-wave interruption. Between 12 and 24 hours there were 46 episodes of sinus $T$-wave interruption and 12 of ventricular $\mathrm{T}$-wave interruption. Only eight further episodes of R-on-T VPB occurred between 24 and 48 hours after the start of the study.

$V P B$ of the R-on-apex-T type-Five patients showed VPB of the $\mathrm{R}$-on-apex-T type: these included $\mathrm{R}$-on-apex-T VPB in which ectopic $R$ waves interrupted the apex of a sinus $T$ wave and those in which ectopic $R$ waves interrupted the apex of ventricular ectopic $T$ waves. In the first 12 hours five sinus $T$-wave interruptions and three ventricular ectopic $T$-wave interruptions occurred. These events continued to appear sporadically, four episodes of sinus $T$-wave 
interruption and four of ventricular ectopic $\mathrm{T}$-wave interruption occurring between 12 and 24 hours, and a further three episodes of sinus $T$-wave interruption occurring between 24 and 48 hours after the study began.

RELATION BETWEEN PLASMA CATECHOLAMINE CONCENTRATIONS AND INCIDENCE OF VENTRICULAR ARRHYTHMIAS

No correlation was found between the number of patients showing ventricular tachycardia or any type of VPB (as described) and the mean catecholamine concentrations (table). Similarly, no correlation could be found between ventricular arrhythmias and maximum plasma catecholamine concentrations.

Relation between maximum and mean plasma catecholamine concentrations and incidence of different ventricular arrhythmias in 26 patients with acute myocardial infarction. Figures are numbers of patients

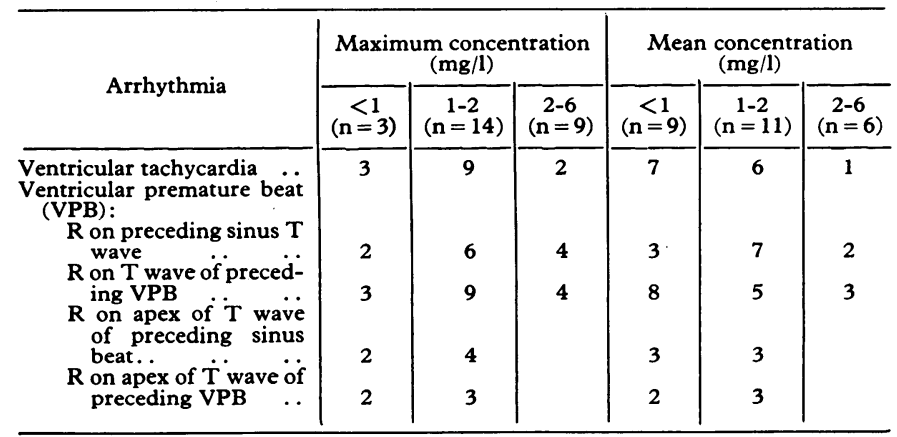

\section{Discussion}

In this study we were unable to identify any relation between the mean or maximum plasma total catecholamine concentration and the incidence of ventricular tachycardia or R-on-T VPB in patients during the first $\mathbf{4 0}$ hours after AMI. In particular, there was no relation between mean or peak catecholamine concentrations and the incidence of R-on-apex-T VPB, which have been said to be precursors of ventricular fibrillation. ${ }^{15}$ Some patients with high mean or peak plasma total catecholamine concentrations did not have any episode of ventricular tachycardia or R-on-T or R-on-apex-T VPB.

The mean plasma total catecholamine concentration for all the patients did not fall significantly and remained at about twice the upper limit of the reference range throughout the study. ${ }^{16}$ The overall incidence of each arrhythmia, however, decreased with time; ventricular tachycardia was not seen after 30 hours from the onset of symptoms, and although R-on- $\mathrm{T}$ and R-on-apex-T VPB were still being recorded at the end of the study, their incidence was much lower after 30 hours. Mean total plasma catecholamine concentrations at this time were about the same as those recorded within the first 12 hours after the onset of symptoms.

The finding of raised plasma catecholamine concentrations after acute myocardial infarction has led to the suggestion that sympathetic stimulation contributes to the genesis of serious ventricular arrhythmias. ${ }^{9}$ Support for this comes from many animal studies - for example, coronary artery ligation in the dog results in a rapid increase in catecholamine efflux from the myocardium, ${ }^{17}$ which commonly precedes the onset of ventricular arrhythmias. ${ }^{1819}$

No conclusion can be reached from the results of this study about the relation between plasma catecholamine concentration and the development of early ventricular arrhythmias, since in only a few patients were the concentrations measured within six hours of the onset of symptoms, and it is during this period that the incidence of ventricular fibrillation, the most serious ventricular arrhythmia, is highest. Nevertheless, there appears to be no relation between plasma catecholamine concentrations and ventricular arrhythmias recorded between about six and 48 hours after the onset of symptoms. Since the vulnerability of the ischaemic myocardium decreases with time, the presencefo of raised plasma catecholamine concentrations several hours? after recovery from acute ischaemia may not have the same ${ }_{0}^{\mathbb{2}}$ effect in inducing re-entry arrhythmias. The time during which most patients were studied-namely, six to 48 hours after the onset of symptoms-probably coincides with the quiescent $\stackrel{\rho^{2}}{+}$ phase (stage II) of arrhythmias seen experimentally, ${ }^{20}$ and it might be expected therefore that $\beta$-adrenergic blocking drugs $\frac{\overline{\bar{N}}}{\mathrm{~N}}$ would not have a profound antiarrhythmic effect at this time. $\frac{\bar{s}}{\bar{\sigma}}$ The results of a multicentre trial of practolol support this $\mathbb{\Phi}$ suggestion in that the reduction in mortality (probably mostly due to arrhythmias) that was achieved during the second infarction $\rightarrow$ was confined to the first two hours after the onset of symptoms. ${ }^{21}$

\section{References}

1 Vetter, N J, et al, Lancet, 1974, 1, 284. ${ }^{1}$ Vetter, N J, et al, Lancet, 1974, 1, 284.
2 Videbaek, J, Christensen, N J, and Sterndorff, B, Circulation, 1972, 46, $\frac{\text { त }}{\mathrm{N}}$
$\quad 846$.

${ }^{3}$ Siggers, D C, Salter, C, and Fluck, D C, British Heart fournal, 1971, 33, 878

${ }^{4}$ Jequier, E, and Perret, C, European fournal of Clinical Investigation, $\overrightarrow{0}$ $1970,1,77$.

5 Valori, C, Thomas, M, and Shillingford, J P, Lancet, 1967, 1, 127.

6 Januszewicz, W, et al, American Heart fournal, 1968, 76, 345.

7 Jewitt, D E, et al, Lancet, 1969, 1, 635.

8 Rosenbaum, M, and Doyle, A E, Australasian Annals of Medicine, 1970, 4, 310 .

${ }^{9}$ McDonald, L, et al, Lancet, 1969, 2, 1021.

10 Rowe, M J, Neilson, J M M, and Oliver, M F, Lancet, 1975, 1, 295.

11 Rose, G A, and Blackburn, H, Cardiovascular Survey Methods. Geneva, WHO, 1968.

12 Smith, A F, Lancet, 1967, 2, 178.

13 Carruthers, M, et al, Lancet, 1970, 2, 62.

14 Neilson, J M M, Institution of Electrical Engineers, conference publicationo No 79, p 151. September 1971.

15 Smirk, F H, and Palmer, D G, American fournal of Cardiology, 1960, 6, 620.

16 Strange, R C, et al, European fournal of Clinical Investigation, 1974, 4, 115. 응

17 Shahab, L, et al, in Effect of Acute Ischaemia on Myocardial Function, ed M F Oliver, D G Julian, and K W Donald, p 97. Edinburgh, Churchill $\overrightarrow{\bar{O}}$ Livingstone, 1972.

18 Staszewska-Barczak, S, and Ceremuzynski, L, Clinical Science, 1968, 34, 531.

19 Ceremuzynski, L, Staszewska-Barczak, S, and Herbaczynska-Cedro, $\mathrm{K}$, Cardiovascular Research, 1969, 3, 190.

${ }^{20}$ Harris, A S, Circulation, 1950, 53, suppl No 1, p 55.

${ }^{21}$ Multicentre International Study, British Medical fournal, 1975, 3, 735.

(Accepted 1 August 1978)

ONE HUNDRED YEARS AGO Dr John Gay of Geelong, Australia, recommends for use in civil and military hospitals, and also for the purpose of destroying the poison-germs of small-pox, scarlet fever, and other infectious diseases, a disinfectant ingeniously com- $N$ posed of one part of rectified oil of turpentine, and seven parts of N benzine, with the addition of five drops of oil of verbena to each ${ }^{\omega}$ ounce. Its purifying and disinfecting properties are due to the power which is possessed by each of its ingredients of absorbing atmospherice oxygen, and converting it into perokide of hydrogen-a highly active oxidising agent, and very similar in its nature to ozone. Articles of ? clothing, furniture, wall-paper, carpeting, books, newspapers, letters, etc, may be perfectly saturated with it without receiving the slightesto injury; and when it has been once freely applied to any rough or porous surface, its action will be persistent for an almost indefinite period. This may, at any time, be readily shown by pouring a few drops of a solution of iodide of potassium over the material which has been disinfected, when the peroxide of hydrogen which is being continually generated within it will quickly liberate the iodine fromo its combination with the potassium, and given rise to dark brown stains. It may be applied with a brush or a sponge, or, if more convenient, as is the case with certain articles, such as books, newspapers; and letters, it may be simply poured over them until they are well soaked; they may then be allowed to dry, either in a warm room or in the open air. (British Medical fournal, 1878.) 